Д. С. Сандитов, А. А. Машанов, С. С. Бадмаев. Нелинейность силы межатомного взаимодействия и упругие свойства твердых тел

УДК 532.138:539.32:666.1

DOI $10.18101 / 2306-2363-2020-2-7-16$

\title{
НЕЛИНЕЙНОСТЬ СИЛЫ МЕЖАТОМНОГО ВЗАИМОДЕЙСТВИЯ И УПРУГИЕ СВОЙСТВА ТВЕРДЫХ ТЕЛ
}

(C) Сандитов Д. С.

доктор физико-математических наук, профессор,

Бурятский государственный университет имени Доржи Банзарова

670000, Улан-Удэ, ул. Смолина, 24а

Институт физического материаловедения СО РАН

670047, Улан-Удэ, ул. Сахьяновой, 6

sanditov@bsu.ru

(C) Машанов А. A.

кандидат технических наук, доцент,

Бурятский государственный университет имени Доржи Банзарова

670000, Улан-Удэ, ул. Смолина, 24а

Mashanov@bsu.ru

(C) Бадмаев C. C.

кандидат технических наук, доцент,

Бурятский государственный университет имени Доржи Банзарова

670000, Улан-Удэ, ул. Смолина, 24а.

sayan75@mail.ru

Установлено, что в формулах Леонтьева и Беломестных-Теслевой для параметра Грюнайзена правые части равенств зависят от ангармонизма через зависимость отношения квадратов скоростей акустических волн $\left(\mathrm{v}_{\mathrm{L}}{ }^{2} / \mathrm{v}_{\mathrm{S}}{ }^{2}\right)$ от параметра Грюнайзена $\gamma$. Теоретическая зависимость $\left(\mathrm{v}_{\mathrm{L}}{ }^{2} / \mathrm{v}_{\mathrm{S}}{ }^{2}\right)$ от $\gamma$ в целом согласуется с экспериментальными данными как для кристаллов, так и для стеклообразных твердых тел. Величина $\left(\mathrm{v}_{\mathrm{L}}{ }^{2} / \mathrm{v}_{\mathrm{S}}{ }^{2}\right)$ оказывается однозначной функцией отношения тангенциальной и нормальной жесткостей межатомной связи.

Ключевые слова: параметр Грюнайзена; продольная и поперечная скорости акустических волн; ангармонизм; коэффициент Пуассона; твердые тела; формулы Леонтьева и Беломестных-Теслевой.

Для цитирования:

Сандитов Д. С., Машанов А. А., Бадмаев С. С. Нелинейность силы межатомного взаимодействия и упругие свойства твердых тел // Вестник Бурятского государственного университета. Химия. Физика. 2020. Вып. 2. С. 7-16.

\section{Введение}

В уравнение состояния твердого тела входит параметр Грюнайзена $\gamma$, характеризующий нелинейность силы межатомного взаимодействия и ангармонизм колебаний решетки. Основным соотношением для экспериментального определения $\gamma$ является уравнение (закон, формула) Грюнайзена [1]

$$
\gamma=\frac{\beta V B}{C_{V}},
$$


где $\beta$ - коэффициент объемного теплового расширения, $V$ - молярный объем, $B$ - изотермический модуль объемного сжатия, $C_{V}$ - молярная теплоемкость при постоянном объеме.

Помимо этого уравнения для расчета $\gamma$ используются другие выражения, в том числе формулы Леонтьева [2]

$$
\gamma=\frac{3}{2}\left(\frac{B_{A}}{\rho v_{\kappa}^{2}}\right)
$$

и Беломестных-Теслевой [3]

$$
\gamma=\frac{3}{2}\left(\frac{1+\mu}{2-3 \mu}\right)
$$

Здесь $B_{A}$ - адиабатический модуль объемного сжатия, $\rho-$ плотность, $v_{\kappa}-$ средняя квадратичная скорость волн деформации, квадрат которой является инвариантом суммы квадратов скоростей распространения продольной $\left(v_{L}\right)$ и поперечной $\left(v_{S}\right)$ упругих волн

$$
v_{\kappa}^{2}=\frac{v_{\mathrm{L}}^{2}+2 v_{\mathrm{S}}^{2}}{3}
$$

$\mu$ - коэффициент Пуассона, который иногда называют коэффициентом поперечной деформации. Формулы Леонтьева (2) и Беломестных-Теслевой (3) привлекательны тем, что в отличие от уравнения Грюнайзена (1) позволяют рассчитывать $\gamma$ по более доступным экспериментальным данным. Установлено, что они находятся в удовлетворительном согласии с уравнением Грюнайзена [3-5] (например, рис. 1).

Вместе с тем обращает внимание то обстоятельство, что в формулах (2) и (3) в левых частях равенств находится мера ангармонизма $\gamma$, а в правые части входят на первый взгляд только гармонические характеристики $\left(\rho, B_{A}, v_{\kappa}^{2}\right)$ и $\mu$. Тем самым наблюдается как бы противоречие.

В настоящем сообщении развито представление о том, что правые части равенств (2) и (3), зависят от ангармонизма (неявно) через зависимость отношения квадратов скоростей звука $\left(v_{\mathrm{L}}^{2} / v_{\mathrm{S}}^{2}\right)$ от параметра Грюнайзена $\gamma$ и поэтому указанное выше противоречие на самом деле является кажущимся противоречием.

\section{Линейная зависимость $\left(v_{1}^{2} / v_{\mathrm{s}}^{2}\right)$ от параметра Грюнайзена}

При изучении формул (2) и (3) обнаруживается тот факт, что их правые части являются функциями отношения квадратов скоростей распространения продольной и поперечной акустических волн $\left(v_{L}^{2} / v^{2}\right)$. Так, например, в уравнении Леонтьева (2) за счет величины $v^{2}$ правая часть равенства оказывается функцией указанного отношения $\left(v_{\mathrm{L}} / v_{\mathrm{S}}\right)^{2}$ (соотношение (4)) 
Д. С. Сандитов, А. А. Машанов, С. С. Бадмаев. Нелинейность силы межатомного взаимодействия и упругие свойства твердых тел

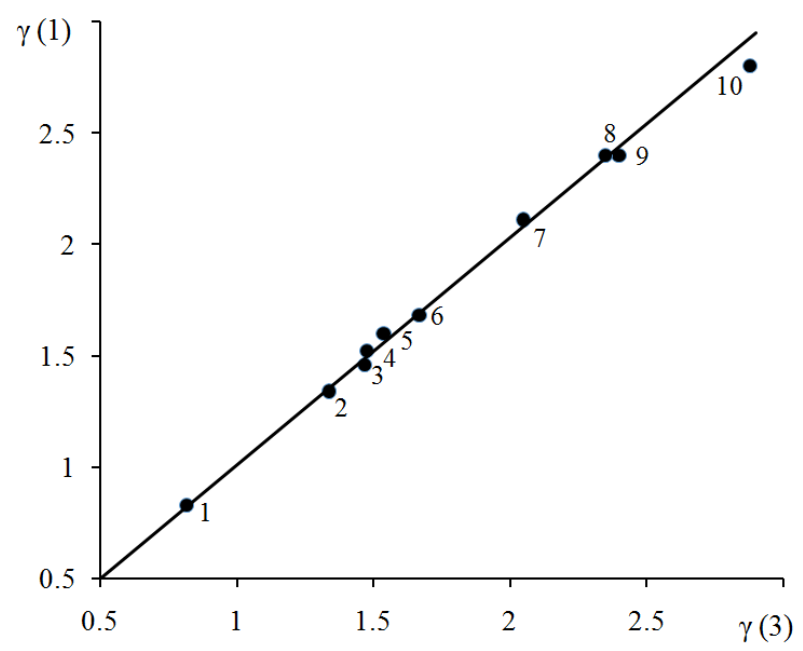

Рис. 1. Линейная корреляция между значениями параметра Грюнайзена $\gamma$, полученными по уравнению Грюнайзена $\gamma(1)$ и по формуле Беломестных-Теслевой $\gamma(3)$, для различных кристаллов (использованы данные [3, 4]).

$1-\mathrm{Be}, 2-\mathrm{LiF}, 3-\mathrm{NaCl}, 4-\mathrm{LiCl}, 5-\mathrm{KCl}, 6-\mathrm{KBr}, 7-\mathrm{Al}, 8-\mathrm{Ag}, 9-\mathrm{Pb}, 10-\mathrm{Au}$.

$$
v_{\kappa}^{2}=\frac{v_{\mathrm{S}}^{2}}{3}\left[\left(\frac{v_{\mathrm{L}}}{v_{\mathrm{S}}}\right)^{2}+2\right] \text {. }
$$

Далее, в правой части уравнения Беломестных-Теслевой (3) коэффициент Пуассона $\mu$, согласно известной формуле теории упругости [6], также является функцией отношения квадратов скоростей звука $\left(v_{L}^{2} / v_{S}^{2}\right)$

$$
\mu=\frac{2-\left(v_{\mathrm{L}} / v_{\mathrm{S}}\right)^{2}}{2-2\left(v_{\mathrm{L}} / v_{\mathrm{S}}\right)^{2}} .
$$

Отмеченное выше наблюдение в отношении рассматриваемых двух формул наводит на мысль о том, что их правые части, возможно, зависят от ангармонизма за счет отношения квадратов скоростей продольной и поперечной акустических волн $\left(v_{L}^{2} / v_{S}^{2}\right)$. В самом деле, наши исследования ряда металлов, ионных и молекулярных кристаллов показали [7]: если между параметром Грюнайзена $\gamma$ и квадратами скоростей $v_{L}^{2}$ и $v_{S}^{2}$ в отдельности фактически нет определенной взаимосвязи (рис. 2 и 3), то их отношение $\left(v_{\mathrm{L}}{ }^{2} / v_{\mathrm{s}}{ }^{2}\right)$. оказывается линейной функцией параметра Грюнайзена $\gamma$ - меры ангармонизма (рис. 4).

У стеклообразных твердых тел наблюдается аналогичная линейная эмпирическая корреляция между отношением квадратов скоростей звуковых волн и параметром Грюнайзена (рис. 5, табл. 2).

\section{Теоретический вариант зависимости $\left(v_{\mathrm{L}}^{2} / v_{\mathrm{s}}^{2}\right)$ от $\gamma$}

На рис. 4 и 5 приводится линейная корреляция между величинами $\left(v_{L}^{2} / v_{S}^{2}\right)$ и $\gamma$, полученная эмпирически на основе экспериментальных данных. Представляет 
интерес установление взаимосвязи этих величин с помощью существующих теоретических уравнений в данной области.

Таблица 1

Скорости звука, коэффициент Пуассона и параметр Грюнайзена неорганических веществ при стандартных условиях $\left(\mathrm{p}=10^{5}\right.$ Па и $\left.\mathrm{T}=298 \mathrm{~K}\right)$

\begin{tabular}{|c|c|c|c|c|c|c|}
\hline \multirow[t]{2}{*}{ № } & \multirow{2}{*}{$\begin{array}{c}\text { Элементы } \\
\text { и соедине- } \\
\text { ния }\end{array}$} & \multicolumn{2}{|c|}{$\begin{array}{c}\text { Скорость } \\
\text { звука, } \\
\text { м/с } \\
\end{array}$} & \multirow{2}{*}{$\begin{array}{c}\text { Отношение } \\
\text { квадратов } \\
\text { скоростей, } \\
\left(v_{\mathrm{L}} / v_{\mathrm{S}}\right)^{2} \\
\end{array}$} & \multirow[t]{2}{*}{$\begin{array}{c}\text { Коэффициент } \\
\text { Пуассона, } \\
\mu\end{array}$} & \multirow[t]{2}{*}{$\begin{array}{c}\text { Параметр } \\
\text { Грюнайзена, } \\
\text { Y }\end{array}$} \\
\hline & & $v_{\mathrm{L}}$ & $v_{\mathrm{S}}$ & & & \\
\hline 1. & $\mathrm{LiF}$ & 7323 & 4518 & 2.627 & 0.200 & 1.34 \\
\hline 2. & $\mathrm{NaCl}$ & 4666 & 2755 & 2.869 & 0.243 & 1.46 \\
\hline 3. & $\mathrm{LiCl}$ & 5260 & 3058 & 2.959 & 0.245 & 1.52 \\
\hline 4. & $\mathrm{NaBr}$ & 3284 & 1885 & 3.35 & 0.270 & 1.56 \\
\hline 5. & $\mathrm{KCl}$ & 4090 & 2312 & 3.130 & 0.259 & 1.60 \\
\hline 6. & $\mathrm{KI}$ & 2623 & 1469 & 3.188 & 0.265 & 1.63 \\
\hline 7. & W & 5233 & 2860 & 3.348 & 0.283 & 1.62 \\
\hline 8. & $\mathrm{Fe}$ & 6064 & 3325 & 3.326 & 0.292 & 1.68 \\
\hline 9. & $\mathrm{KF}$ & 4641 & 2587 & 3.218 & 0.274 & 1.73 \\
\hline 10. & $\mathrm{Rbl}$ & 2245 & 1198 & 3.512 & 0.309 & 1.73 \\
\hline 11. & Co & 5827 & 3049 & 3.652 & 0.357 & 1.87 \\
\hline 12. & $\mathrm{Cu}$ & 4726 & 2298 & 4.229 & 0.350 & 2.00 \\
\hline 13. & $\mathrm{Ag}$ & 3686 & 1677 & 4.831 & 0.379 & 2.40 \\
\hline 14. & $\mathrm{Pt}$ & 3960 & 1670 & 5.623 & 0.390 & 2.54 \\
\hline 15. & $\mathrm{~Pb}$ & 2158 & 860 & 6.30 & 0.372 & 2.93 \\
\hline
\end{tabular}

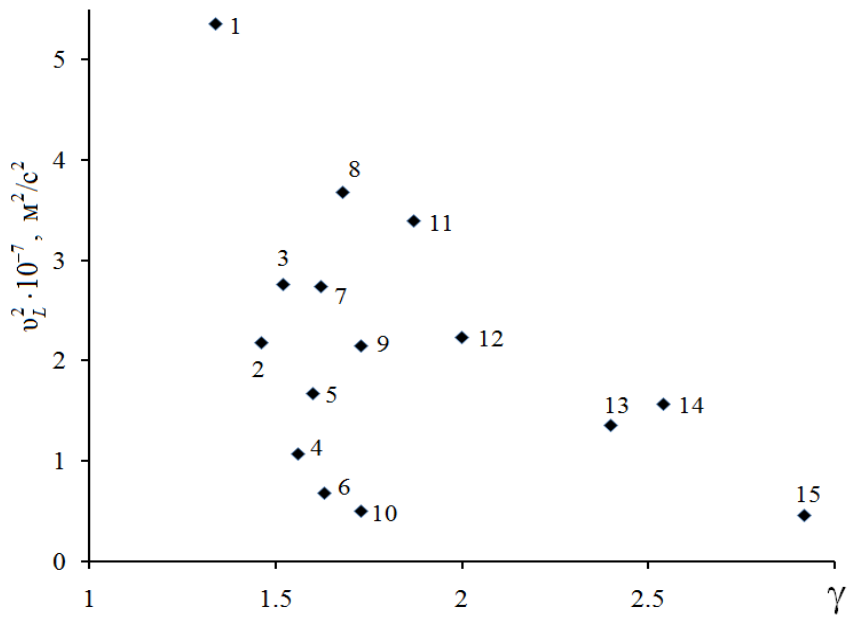

Рис. 2. Зависимость квадрата скорости продольной акустической волны ${ }^{2}$ от параметра Грюнайзена $\gamma$ для ряда кристаллов. Номера точек соответствуют номерам веществ в табл. 1. 
Д. С. Сандитов, А. А. Машанов, С. С. Бадмаев. Нелинейность силы межатомного взаимодействия и упругие свойства твердых тел

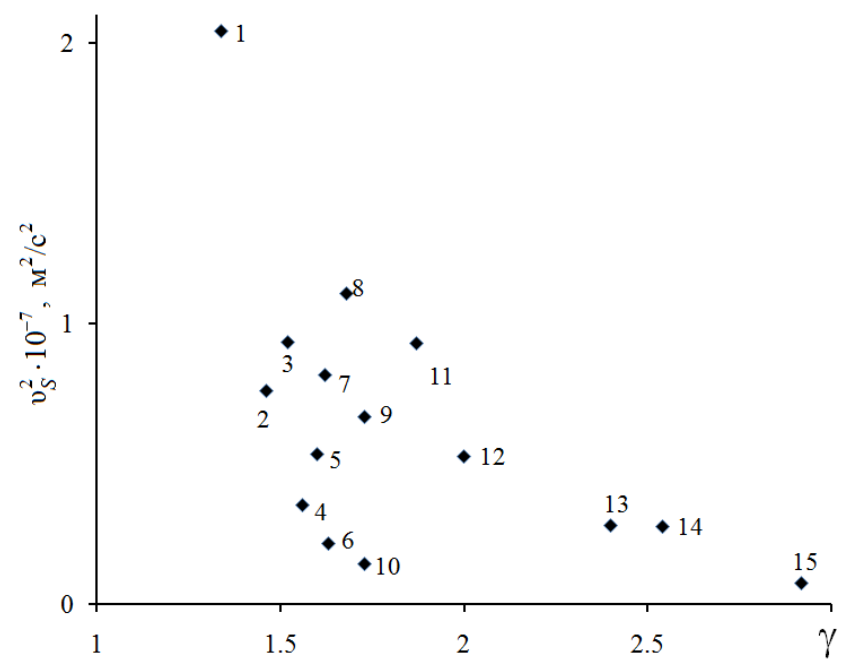

Рис. 3. Зависимость квадрата скорости поперечной акустической волны ${ }_{s}^{2}$ от параметра Грюнайзена $\gamma$ для кристаллов, приведенных на рис. 2. Номера точек соответствуют номерам веществ в табл. 1.

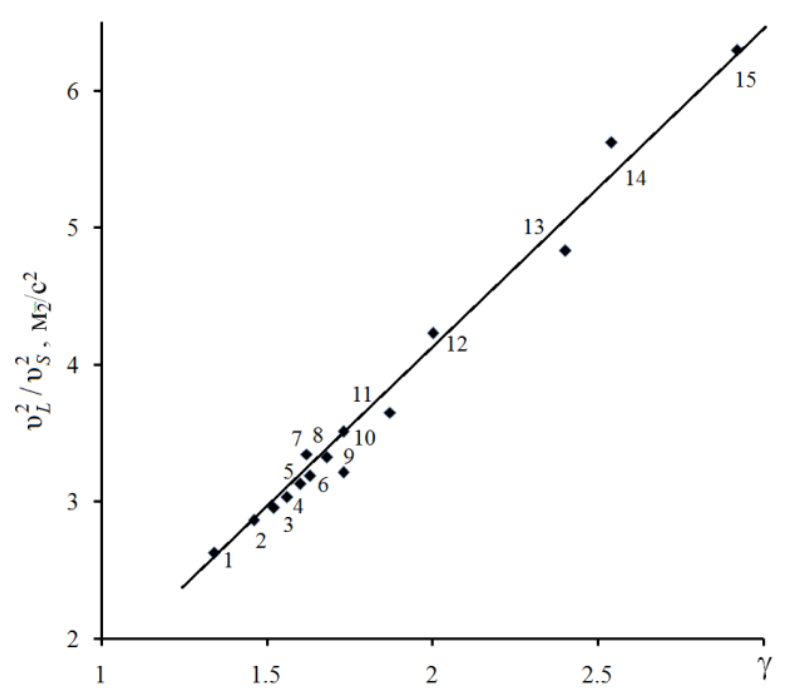

Рис. 4. Линейная корреляция между отношением квадратов продольной и поперечной скоростей звука $\left(v_{\mathrm{L}}{ }^{2} / v_{\mathrm{s}}{ }^{2}\right)$ от параметра Грюнайзена $\gamma$ для кристаллов, приведенных на рис. 2 и 3. Номера точек соответствуют номерам веществ в табл.1. 


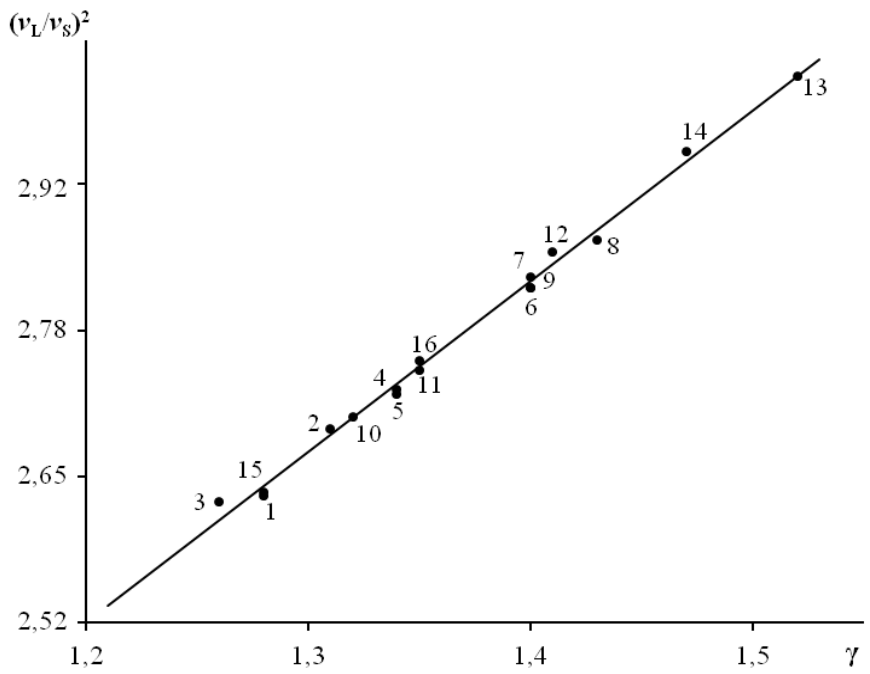

Рис. 5. Линейная корреляция между отношением квадратов скоростей распространения акустических волн $\left(v_{\mathrm{L}} / v_{\mathrm{S}}\right)^{2}$ и параметром Грюнайзена $\gamma$. Натриевоалюмосиликатные стекла $\mathrm{Na}_{2} \mathrm{O}-\mathrm{Al}_{2} \mathrm{O}_{3}-\mathrm{SiO}_{2}$ с разным содержанием окислов. Номера точек соответствуют номерам стекол в табл. 2.

Таблица 2

Плотность $\rho$, скорости распространения продольных $\left(v_{\mathrm{L}}\right)$ и поперечных $\left(v_{\mathrm{S}}\right)$ акустических волн, модуль объемного сжатия $B_{A}$, коэффициента Пуассона $\mu$ и параметр Грюнайзена $\gamma$ для стекол $\mathrm{Na}_{2} \mathrm{O}-\mathrm{Al}_{2} \mathrm{O}_{3}-\mathrm{SiO}_{2}$

(Использованы данные [8])

\begin{tabular}{|c|c|c|c|c|c|c|c|c|c|}
\hline \multirow[t]{2}{*}{ № } & \multicolumn{3}{|c|}{$\begin{array}{c}\text { Состав по синтезу, } \\
\text { мол. \% }\end{array}$} & \multirow[t]{2}{*}{$\rho \cdot 10^{-3}, \mathrm{kr} / \mathrm{M}^{3}$} & \multirow{2}{*}{$\begin{array}{l}V_{L \prime} \\
M / C\end{array}$} & \multirow{2}{*}{$\begin{array}{l}v_{S}, \\
M / C\end{array}$} & \multirow{2}{*}{$\begin{array}{c}B_{A} \cdot 10^{-8}, \\
\text { Па }\end{array}$} & \multirow[t]{2}{*}{$\mu$} & \multirow[t]{2}{*}{$\gamma$} \\
\hline & $\mathrm{Na}_{2} \mathrm{O}$ & $\mathrm{Al}_{2} \mathrm{O}_{3}$ & $\mathrm{SiO}_{2}$ & & & & & & \\
\hline 1 & 15 & 0 & 85 & 2339 & 5430 & 3340 & 342 & 0.196 & 1.28 \\
\hline 2 & 15 & 5 & 80 & 2358 & 5570 & 3390 & 370 & 0.206 & 1.31 \\
\hline 3 & 15 & 10 & 75 & 2410 & 5697 & 3510 & 386 & 0.194 & 1.26 \\
\hline 4 & 15 & 15 & 70 & 2465 & 5737 & 3469 & 416 & 0.212 & 1.34 \\
\hline 5 & 15 & 20 & 65 & 2428 & 5850 & 3540 & 425 & 0.211 & 1.34 \\
\hline 6 & 15 & 25 & 60 & 2472 & 6000 & 3568 & 470 & 0.226 & 1.40 \\
\hline 7 & 25 & 0 & 75 & 2439 & 5280 & 3140 & 359 & 0.226 & 1.40 \\
\hline 8 & 25 & 5 & 70 & 2455 & 5480 & 3240 & 394 & 0.231 & 1.41 \\
\hline 9 & 25 & 10 & 65 & 2461 & 5610 & 3330 & 411 & 0.228 & 1.40 \\
\hline 10 & 25 & 20 & 55 & 2470 & 5680 & 3450 & 405 & 0.208 & 1.32 \\
\hline 11 & 25 & 25 & 50 & 2499 & 5790 & 3490 & 432 & 0.215 & 1.35 \\
\hline 12 & 25 & 30 & 45 & 2519 & 6026 & 3556 & 490 & 0.233 & 1.43 \\
\hline 13 & 35 & 0 & 65 & 2497 & 5340 & 3070 & 398 & 0.253 & 1.52 \\
\hline 14 & 30 & 5 & 65 & 2486 & 5500 & 3200 & 413 & 0.244 & 1.47 \\
\hline 15 & 20 & 15 & 65 & 2450 & 5670 & 3490 & 390 & 0.195 & 1.28 \\
\hline 16 & 17.5 & 17.5 & 65 & 2447 & 5746 & 3458 & 418 & 0.216 & 1.35 \\
\hline
\end{tabular}


Д. С. Сандитов, А. А. Машанов, С. С. Бадмаев. Нелинейность силы межатомного взаимодействия и упругие свойства твердых тел

Формулу для зависимости отношения скоростей звука $\left(v_{L}^{2} / v^{2}\right)$ от параметра Грюнайзена $\gamma$ можно вывести из двух экспериментально оправданных соотношений, а именно из уравнения Беломестных-Теслевой (3) и формулы теории упругости (5), связывающей квадраты скоростей акустических волн с коэффициентом Пуассона $\mu$, которую разрешим относительно $\left(v_{\mathrm{L}}^{2} / v_{\mathrm{S}}^{2}\right)$ и запишем в виде [6]

$$
\left(\frac{v_{\mathrm{L}}}{v_{\mathrm{S}}}\right)^{2}=\frac{2-2 \mu}{1-2 \mu}
$$

Выразив из уравнения Беломестных-Теслевой (3) коэффициент Пуассона $\mu$ через $\gamma$ и подставив его в формулу теории упругости (6), приходим к следующей зависимости отношения $\left(v_{L}^{2} / v^{2}\right)$ от $\gamma$

$$
\left(\frac{v_{\mathrm{L}}}{v_{\mathrm{S}}}\right)^{2}=4\left(\frac{3+\gamma}{9-2 \gamma}\right)
$$

Такой же результат можно получить также из формулы Беломестных для акустического параметра Грюнайзена (соотношение (1) в работе [3]).

Теоретическая зависимость (7) находится в согласии с экспериментальными данными для стекол - прямая на графике проходит практически через начало координат с тангенсом угла наклона, равным единице (рис. 6). Исследованные кристаллы (табл. 1) в целом подчиняются зависимости (7), однако они по отношению к ней делятся на две группы, каждая из которых описывается уравнением прямой, не проходящей через начало координат (рис. 7, a, b),

$$
\left(\frac{v_{\mathrm{L}}}{v_{\mathrm{S}}}\right)^{2}=a\left(\frac{3+\gamma}{9-2 \gamma}\right)+b
$$

где $a$ и $b-$ постоянные $(a \neq 4$ и $b \neq 0)$

Возникает, естественно, вопрос, как согласовать соотношение (7) с эмпирической линейной корреляцией, наблюдаемой между величинами $\left(v_{L}^{2} / v_{S}^{2}\right)$ и $\gamma$ (рис. 4 и 5.). Из формулы (7) можно получить приближенную линейную зависимость $\left(v_{\mathrm{L}}^{2} / v^{2}\right)$ от $\gamma$ при условии $2 \gamma<<9$

$$
\left(\frac{v_{\mathrm{L}}}{v_{\mathrm{S}}}\right)^{2} \approx 1.3+0.4 \gamma
$$

Если для рассмотренных стекол, у которых $\gamma \approx 1.2-1.5$ (табл. 2), данное условие более или менее приемлемо, то для исследованных кристаллов (табл. 1) оно выполняется с натяжкой. Этот вопрос требует дальнейшего исследования.

Заметим, что согласно формуле (7), соблюдается условие $(9-2 \gamma)>0$, откуда $\gamma<4.5$, что совпадает с максимальным параметром Грюнайзена при полиморфных превращениях в кристаллах [9]. 


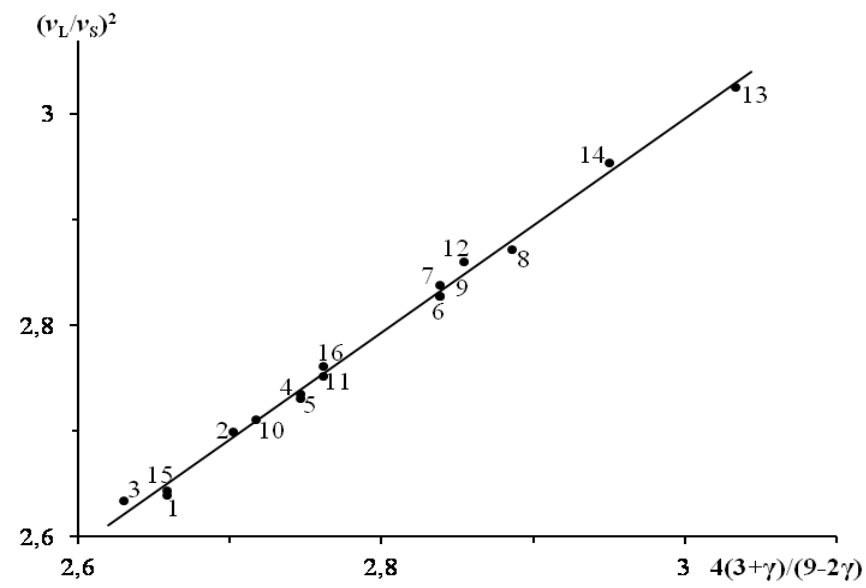

Рис. 6. Зависимость квадрата отношения скоростей распространения продольной $\left(v_{\mathrm{L}}\right)$ и поперечной $\left(v_{\mathrm{S}}\right)$ акустических волн $\left(v_{\mathrm{L}} / v_{\mathrm{S}}\right)^{2}$ от параметра Грюнайзена $\gamma$ в координатах, соответствующих уравнению (7). Натриевоалюмосиликатные стекла $\mathrm{Na}_{2} \mathrm{O}-\mathrm{Al}_{2} \mathrm{O}_{3}-\mathrm{SiO}_{2}$ с разным содержанием окислов. Номера точек соответствуют номерам стекол в табл. 2.

С точки зрения интерпретации полученных результатов на микроскопическом уровне представляет определённый интерес модель случайно упакованных атомов в виде сфер, взаимодействующих друг с другом в месте контакта двумя взаимно перпендикулярными силами нормальной к плоскости контакта $f_{n}=k_{n} x_{n}$ и тангенциальной (силой трения) $f_{t}=k_{t} x_{t}[10]$. В рамках данной модели БерлинаРотенбурга-Басэрста (БРБ) коэффициент Пуассона $\mu$ определяется отношением тангенциальной $k_{t}$ и нормальной $k_{n}$ жесткостей межатомной связи $\lambda=\left(k_{t} / k_{n}\right)[10]$
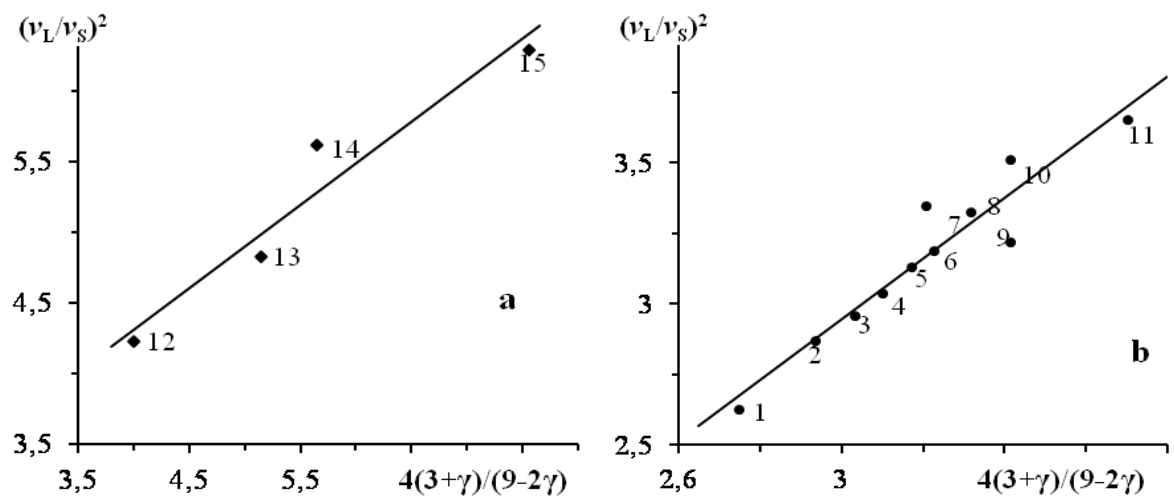

Рис. 7. Зависимость $\left(v_{\mathrm{L}} / v_{\mathrm{S}}\right)^{2}$ от параметра Грюнайзена $\gamma$ в координатах, соответствующих уравнению (7). Номера точек соответствуют номерам кристаллов в табл. 1.

$$
\mu=\frac{1-\lambda}{4+\lambda}
$$

Из соотношений (6) и (9) следует, что отношение квадратов скоростей $\left(v^{2}{ }_{\mathrm{L}} / v_{\mathrm{S}}^{2}\right)$ определяется микроскопическим параметром $\lambda$ 
Д. С. Сандитов, А. А. Машанов, С. С. Бадмаев. Нелинейность силы межатомного взаимодействия и упругие свойства твердых тел

$$
\left(\frac{v_{L}}{v_{S}}\right)^{2}=\frac{2(3+\lambda)}{2+3 \lambda}
$$

В свою очередь, как видно из равенств (3) и (9), параметр $\lambda$ однозначно связан с ангармонизмом $(\gamma)$.

\section{Заключение}

Таким образом, квадраты скоростей продольной и поперечной акустических волн $v_{L}^{2}$ и $v_{S}^{2}$ в отдельности практически не связаны с ангармонизмом - не коррелируют с параметром Грюнайзена, а их отношение $\left(v_{L}^{2} / v_{S}^{2}\right)$ оказывается ангармонической (нелинейной) характеристикой твердых тел. В формуле Леонтьева (2) и Беломестных-Теслевой (3) нет противоречия, касающегося взаимосвязи гармонических и ангармонических величин. Как левые, так и правые части равенств в этих соотношениях зависят от нелинейности силы межатомного взаимодействия - ангармонизма, мерой которого служит параметр Грюнайзена $\gamma$. Отношение $\left(v_{\mathrm{L}}^{2} / v_{\mathrm{S}}^{2}\right)$ определяется отношением тангенциальной и нормальной жесткостей межатомной связи $\lambda=\left(k_{t} / k_{n}\right)$, которое является однозначной функцией параметра Грюнайзена.

\section{Литература}

1. Жирифалько Л. Статистическая физика твердого тела. - М.: Мир, 1975. - 382 с.

2. Леонтьев К. Л. О связи упругих и тепловых свойств веществ // Акуст. журн. 1981. - Т. 27, Вып. 4. - С. 554-561.

3. Беломестных В. Н., Теслева Е. П. Взаимосвязь ангармонизма и поперечной деформации квазиизотропных поликристаллических тел // ЖТФ. — 2004. - Т. 74, Вып. 8. С. $140-142$.

4. Сандитов Д. С., Беломестных В. Н. Взаимосвязь параметров теории упругости и усредненный модуль объемного сжатия твердых тел // ЖТФ. — 2011. - Т. 81, Вып. 11. - C. 77-81.

5. Сандитов Д. С. Природа коэффициента Пуассона аморфных полимеров и стекол и его связь со структурно-чувствительными свойствами // УФН. — 2020. — Т. 190, № 4. C. $355-370$.

6. Ландау Л. Д., Лифшиц Е. М. Теория упругости. — М.: Наука, 1965. — 204 с.

7. Сандитов Д. С., Дармаев М. В.,Сандитов Б. Д., Мантатов В. В. Параметр Грюнайзена и скорости распространения звуковых волн в твердых телах // Изв. вузов. Физика. 2009. — № 4. - С. 50-52.

8. Лившиц В. Я., Теннисон Д. Г., Гукасян С. Б., Костанян А. К. Акустические и упругие свойства стекол системы $\mathrm{Na}_{2} \mathrm{O}-\mathrm{Al}_{2} \mathrm{O}_{3}-\mathrm{SiO}_{2} / /$ ФХС. - 1982. — Т. 8, № 6. - С. 688693.

9. Беломестных В. Н., Теслева Е. П., Соболева Э. Г. Максимальный параметр Грюнайзена при полиморфных превращениях в кристаллах // ЖТФ. — 2009. — Т. 79, Вып. 2. — C. $153-154$.

10. Берлин А. А., Ротенбург Л., Басэрст Р. Структура изотропных материалов с отрицательным коэффициентом Пуассона // Высокомолек. соединения. Сер. Б. - 1991. T. 33, № 8. - С. 619-621 (Обзор). 


\section{NONLINEARITY OF THE INTER-ATOMIC INTERACTION FORCE} AND ELASTIC PROPERTIES OF SOLIDS

Sanditov D. S.

Doctor of Physical and Mathematical Sciences, Professor, Banzarov Buryat State University 670000, Ulan-Ude, Smolina str., 24a Institute of Physical Materials Science SB RAS 670047 Ulan-Ude, ul. Sakhyanova, 6 sanditov@bsu.ru.

Mashanov A. A.

Candidate of Technical Sciences, associate Professor, Banzarov Buryat State University 670000, Ulan-Ude, Smolina str., 24a.

Mashanov@bsu.ru.

Badmaev S. S.

Candidate of Technical Sciences, associate Professor, Banzarov Buryat State University 670000, Ulan-Ude, Smolina str., 24a sayan75@mail.ru

It was found that in the Leontiev and Belomestnykh-Tesleva formulas for the Grüneisen parameter, the right-hand sides of the equalities depend on anharmonicity through the dependence of the ratio of the squared acoustic wave velocities $\left(\mathrm{v}_{\mathrm{L}}^{2} / \mathrm{v}_{\mathrm{S}}^{2}\right)$ on the Grüneisen parameter $\gamma$. The theoretical dependence of $\left(\mathrm{v}_{\mathrm{L}}^{2} / \mathrm{v}_{\mathrm{S}}{ }^{2}\right)$ on $\gamma$ generally agrees with experimental data for both the crystals and glassy solids. The quantity $\left(\mathrm{v}_{\mathrm{L}}{ }^{2} / \mathrm{v}_{\mathrm{S}}{ }^{2}\right)$ turns out to be a single-valued function of the ratio of the tangential and normal stiffness of the interatomic bond.

Keywords: Grüneisen parameter; longitudinal and transverse velocities of acoustic waves; anharmonicity; Poisson's ratio; solids; Leontiev and Belomestnykh-Tesleva formulas.

Статья поступила в редакцию 23.09.2020; принята к публикации 30.10.2020. 